\title{
BASES PARA LA CONFECCIÓN DEL ATLAS FLORÍSTICO DE LA PROVINCIA DE MÁLAGA
}

\author{
A. M. Hernández, A. Asensi y B. Díez*
}

\section{RESUMEN}

Se examinan los atlas florísticos hechos últimamente en Europa, especialmente los que siguen la cuadrícula UTM, y se comentan las características de cada uno de ellos. A continuación se señalan los criterios que se han seguido para la elaboración del plano matriz, en cuadrícula UTM, para la provincia de Málaga, y los trabajos que se siguen, a fin de hacer el correspondiente atlas florístico.

El estudio corológico detallado para las plantas vasculares comenzó en Europa con el trabajo de Hultén (1950) Atlas över vaxternas utbredning $i$ norden, donde se representa gráficamente, a escala 1:20 000 000, la distribución de las especies cormofíticas de la Escandinavia y de la Fenoscandia, en círculos de $0,8 \mathrm{~mm}$ de diámetro, equivalente a $16 \mathrm{~km}$, los cuales son sustituidos por trazos continuos cuando los táxones son comunes en una determinada área.

En los últimos años se ha generalizado el sistema de proyección cartográfico UTM (Universal Transverse Mercator), sobre todo después que la OTAN cartografiara en la década de 1950 toda la Europa occidental en este sistema, con fines militares, y la posterior aplicación de este sistema en cartografía civil y científica, debido a su facilidad de manejo, precisión, decimalización, etc., y a pesar de los inconvenientes que presenta (áreas donde no es posible establecer cuadrados de $10 \mathrm{~km}$ de lado, sino superficies trapezoidales de menor área).

Así, en 1962, aparece publicado el monumental y preciso Atlas of the British Flora (PERRING y WalTERS, 1962), donde se ha seguido escrupulosamente el cuadriculado UTM. La realización de esta monumental obra ha sido posible por el gran conocimiento que se tenía de la flora británica, por el elevado número de sociedades científicas, especialistas y aficionados que se aprestaron a colaborar en tan magno trabajo, y por la utilización de los modernos sistemas de procesamiento de datos. Los mapas, que incluyen Gran Bretaña, Irlanda e islas adyacentes, están impresos a escala

(*) Departamento de Botánica de la Universidad de Málaga/Instituto Botánico de Málaga. 
1:8 000000 , y en cada uno de ellos se indica el estado de conocimiento de la distribución de la especie en cuestión, si la planta es nativa o no, si los datos son anteriores o posteriores a 1930, etc. Además se incluyen unos mapas superponibles de plástico transparente donde están representados los ríos, comarcas, altitudes, naturaleza geológica del sustrato, temperaturas mínimas y medias de febrero, temperaturas medias de julio y cuadrícula UTM.

Alejándose de esta pauta, y quizá por no querer pasar el cúmulo de datos disponibles al sistema UTM, se han publicado algunos otros trabajos ajenos a este sistema, entre los cuales destaca el laboriosísimo Atlas de la flore belge et luxemburgeoise (Rompaey y Delvosalle, 1972), que utiliza una red de cuadrados de $16 \mathrm{~km}^{2}$, siendo poco manejable y no homologable.

En 1964, a raíz del X Congreso Internacional de Botánica, celebrado en Edimburgo, se pensó en la posibilidad de realizar un atlas de la flora europea. Tras unos ensayos efectuados por F. H. PERring, esta idea cuajó al año siguiente, en el IV Simposio de Flora Europaea, que tuvo lugar en Århus, Dinamarca, y del que nació el Comité para la Cartografía de la Flora Europea, cuyo secretariado asumió J. Suominen. Actualmente está publicado el primero y segundo volumen del Atlas florae Europaeae (JaLAS y Suominen, 1972 y 1973). En esta obra se sigue el cuadriculado UTM, pero tomándose cuadrados de $50 \mathrm{~km}$ de lado. Los mapas son impresos a una escala aproximada de 1:31 000000 , pero hay un mapa matriz de escala 1:10000 000. Para cada especie se da un símbolo, según sea el tipo de cita: plantas nativas, introducidas, citas dudosas, extinciones, etc. Esta gran obra, aunque imperfecta debido al desconocimiento que se tiene de muchas regiones europeas, entre ellas una gran parte de España, servirá de cañamazo para el mejor conocimiento de la corología de las especies europeas. Su realización es posible gracias a: la existencia de atlas florísticos regionales, la aparición de Flora Europaea, la generalización del sistema de proyección cartográfica UTM, y el uso de sistemas electrónicos de procesamiento de datos. En esta obra colaboran los botánicos españoles E. F. Galiano y B. Valdés, del Departamento de Botánica de la Universidad de Sevilla, y P. Montserrat, del Centro Pirenaico de Biología Experimental, de Jaca.

En España, aparte de la colaboración de investigadores españoles en el atlas de la flora europea, se llevan a cabo diversos trabajos en esta línea. Así, el Departamento de Botánica de la Facultad de Biología de la Universidad de Barcelona está elaborando desde hace años el atlas florístico de los Países Catalanes, siguiendo la cuadrícula UTM. Por otra parte, el Departamento de Botánica de la Universidad de Sevilla lleva a cabo la ejecución de los catálogos de la flora vascular de las provincias de Sevilla y de Cádiz, mientras que el Departamento de Botánica de la Universidad de Málaga realiza el catálogo florístico de la provincia de Málaga, consignándose los datos de los tres catálogos en el sistema UTM. Otros diversos trabajos, entre ellos varias tesis doctorales, llevan asimismo los datos expresados en UTM. También, en por lo menos tres herbarios españoles (BC, Herb. JACA y Herb. MÁLAGA), se consigna el cuadrado UTM en las fichas de los nuevos pliegos. 
La provincia de Málaga, que abarca una superficie de $7.276 \mathrm{~km}^{2}$, cae totalmente dentro de la zona UTM 30S. El criterio que hemos seguido para la confección del plano matriz, que se presenta en la figura 1 , ha sido, para la línea de costa, incluir los cuadrados que comprenden más de un $10 \%$ de tierra firme, pero en las divisiones provinciales hemos seguido un criterio más laxo, puesto que los cuadrados que no incluimos en la provincia de Málaga se incluirán forzosamente en las provincias vecinas. No obstante, hemos tomado todos aquellos cuadrados que contuvieran no menos del $20 \%$ de territorio provincial malagueño. Para la realización del mapa matriz, hemos trabajado con las hojas 4-11, 4-12 y 5-11 del Mapa militar de España E: 1:200 000 (SERvicio Cartográfico DEL EJÉrCito, 1968-69).

De acuerdo con lo expuesto, el cuadrado costero UF02 se anexa al UF03; el UF43 al UF44; el UF64 al UF65, y el UF75 al UF76. Los cuadrados comunes con las provincias vecinas, y que incluimos en la de Málaga, son los que a continuación se consignan: con la provincia de Cádiz, los cuadrados TF64, TF74, TF83, TF84, TF85, TF92, TF96, TF97, TF98, UF07, UF18 y UF19; con la provincia de Sevilla, los cuadrados UF19, UG20, UG31, UG41, UG42 y UG52; con la provincia de Córdoba, los cuadrados UG52, UG72 y UG81, y con la provincia de Granada, los cuadrados UF89, UF99, UG80, UG81, VF08, VF18 y VF27. No se incluyen, según el criterio seguido, y por pertenecer en más de un $80 \%$ a la provincia adyacente, los cuadrados TF75 y UF08, colindantes con la provincia de Cádiz; los UG10 y UG21, comunes con la provincia de Sevilla; el UG62, compartido con la provincia de Córdoba, y los VF28, VF36 y VF37, fronterizos con la provincia de Granada.

En total, el mapa matriz de la provincia de Málaga en cuadrícula UTM (véase fig. 1) consta de 90 cuadrados de $100 \mathrm{~km}^{2}$, los cuales están debidamente señalados con sus letras y sus números de clave. También se han establecido unas rayas y unas separaciones que delimitan los cuadrados de $100 \mathrm{~km}$ de lado correspondiente a TF, UF, UG y VF. Asimismo, en la parte media de estas rayas se señala con un trazo perpendicular los cuadrados de $50 \mathrm{~km}$ de lado usados en el atlas de la flora europea, que se designan según el esquema ${ }_{2}^{1} \mathrm{AB}_{4}^{3}$. Así, pues, corresponden a la provincia de Málaga los cuadrados de flora europea TF3, TF4, UF1, UF2, UF3, UG2, UG4 y VF1. El cuadrado de $10 \mathrm{~km}$ de lado UF54 se incorpora al cuadrado de $50 \mathrm{~km}$ de lado UF3.

Debido al número relativamente pequeño de cuadrados a estudiar (90), a las pocas citas existentes en bibliografía y en herbarios (salvo el Herb. MÁLAGA), y a la dificultad de procesar datos de manera continua pero lenta, seguimos para la elaboración del atlas un sistema de ficheros. Para cada especie y para cada cuadrado se abre una ficha, donde se consignan las diversas citas (de herbario, bibliográficas y visuales). Cuando el número de citas fiables de una especie en un cuadrado determinado es suficiente, y si no hay dificultades taxonómicas, se cierra la ficha (excepto para las citas de herbario y bibliográficas) y el dato se consigna definitivamente en fichas impresas con el plano matriz, cuya reproducción se da en la figura 1 . 
La tarea de cartografiar la totalidad de la flora malacitana es ardua y, a pesar del mucho trabajo ya realizado, habrán de transcurrir algunos años antes de que esté totalmente concluida, máxime teniendo en cuenta que no se dispone de ninguna flora provincial completa. Es por ello por lo que el Departamento de Botánica de la Universidad de Málaga, en colaboración con el Instituto Botánico de Málaga, está elaborando el catálogo florístico de la provincia de Málaga, que servirá de base para la realización del atlas florístico y de la flora de la provincia de Málaga.

\section{BIBLIOGRAFIA}

Hultén, E. 1950. Atlas över vaxternas utbredning $i$ norden (Atlas of the distribution of vascular plants in northwestern Europe). Estocolmo. (2." edición 1971).

JAGER, E. y WeINERT, E. 1964. Vergleichende Chorologie der Zentraleuropäischen Flora. Jena.

Jalas, J. y Suominen, J. (ed.). 1972. Atlas florae Europaeae. 1. Pterydophyta. Helsinki.

Jalas, J. y Suominen, J. (ed.) 1973. Atlas florae Europaeae. 2. Gymnospermae. Helsinki.

Perring, F. H. y Walters, S. M. (ed.) 1962. Atlas of the British flora. Londres.

Rompaey, E. Van y Delvosalle, L. 1972. Atlas de la flore belge et luxemburgeoise. Pteridophytes et Spermatophytes. Bruselas.

Servicio Cartográfico Del Ejército. 1968-69. Mapa militar de España E. 1: 200000 (Hojas 4-11, Morón de la Frontera; 4-12, Algeciras, y 5-11, Granada-Málaga). Madrid.

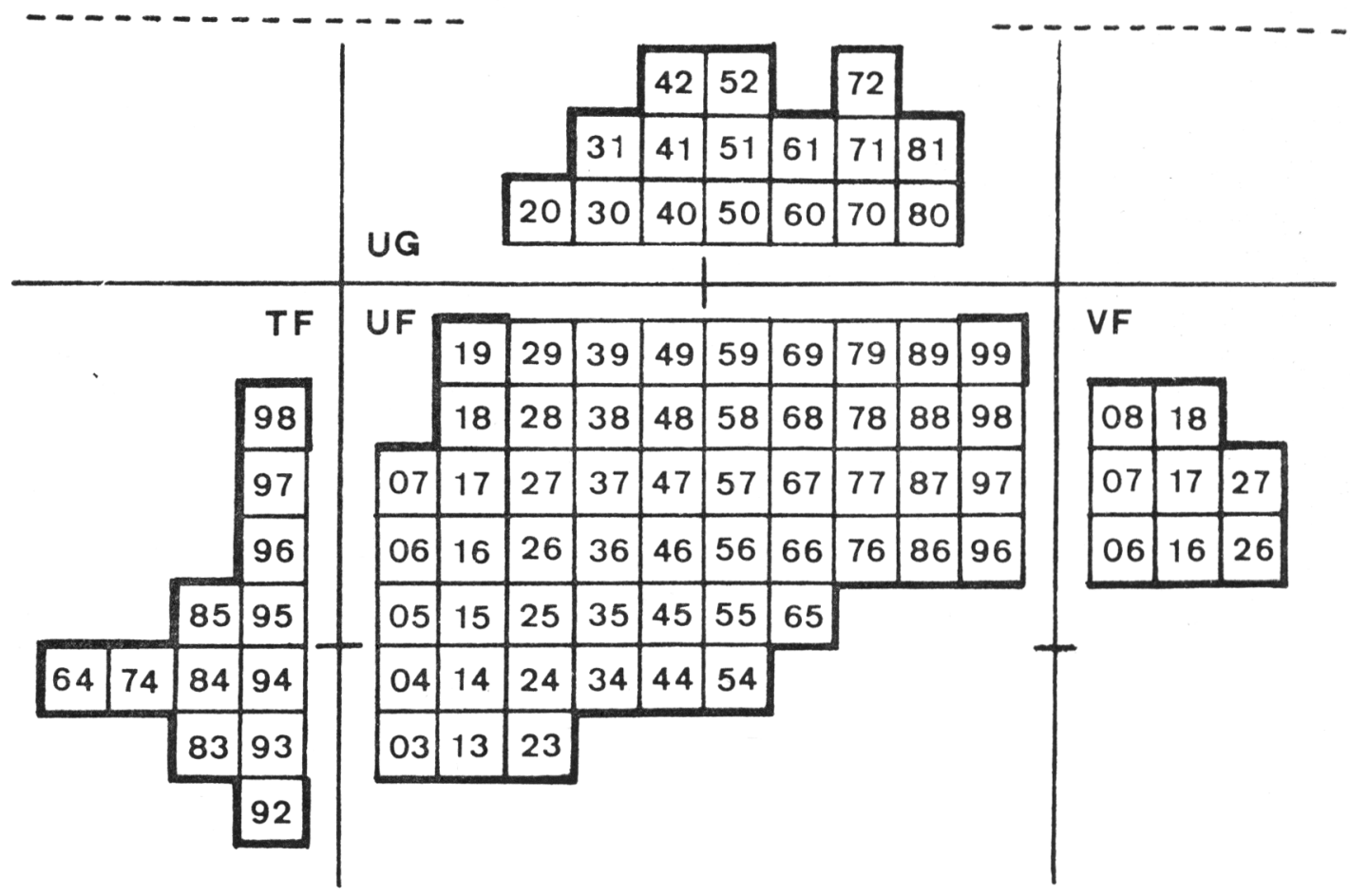

